\title{
A clinical trial report of autologous bone marrow-derived mesenchymal stem cell transplantation in patients with spinal cord injury
}

\author{
PU-CHA JIANG $^{1 *}$, WEN-PING XIONG ${ }^{1 *}$, GE WANG $^{2}$, CHAO MA $^{1}$, WEI-QI YAO ${ }^{2}$, \\ STEVEN F. KENDELL ${ }^{4}$, BRIAN M. MEHLING ${ }^{4}$, XIAN-HOU YUAN ${ }^{5}$ and DONG-CHENG WU ${ }^{2,3}$ \\ ${ }^{1}$ Department of Neurosurgery, Zhongnan Hospital, Wuhan University; ${ }^{2}$ Biochemistry Institute, Wuhan University; \\ ${ }^{3}$ Department of Stem Cells, Wuhan Hongqiao Brain Hospital, Wuhan, Hubei 430071, P.R. China; \\ ${ }^{4}$ Blue Horizon International, LLC, New York, NY 10014, USA; ${ }^{5}$ Department of Neurosurgery, \\ Zhongnan Hospital of Wuhan University, Wuhan, Hubei 430071, P.R. China
}

Received January 18, 2013; Accepted March 27, 2013

DOI: $10.3892 /$ etm.2013.1083

\begin{abstract}
Spinal cord injury (SCI) is a severe neurological disease. An effective strategy for the treatment of SCI is urgently required. Stem cell transplantation has emerged as a viable therapeutic option with great potential for restoring neurological function lost following SCI. From 2009 to 2010, a total of 20 SCI patients were enrolled in a clinical trial by Wuhan Hongqiao Brain Hospital; all patients completed and signed informed consent prior to autologous bone marrow-derived mesenchymal stem cell transplantation. Analysis of subsequent treatment results indicated significant improvements in sensory, motor and autonomic nerve function as assessed by the American Spinal Injury Association's impairment scale. Thirty days after transplantation, a total of 15 patients $(75 \%)$ demonstrated improvement, including four of the eight patients $(50 \%)$ with grade A SCI, three of the four patients $(75 \%)$ with grade B injury and all eight patients $(100 \%)$ with grade $\mathrm{C}$ injury. The most common adverse events, fever and headache, disappeared within 24-48 h without treatment.
\end{abstract}

\section{Introduction}

The repercussions of spinal cord injury (SCI), regardless of origin, are often severe and include loss of bowel and bladder faculties, impairments in sensory and motor function and

Correspondence to: Professor Dong-Cheng Wu, Biochemistry Institute, Wuhan University, Donghu Road, Wuhan, Hubei 430071, P.R. China

E-mail: xianhouyuan@yeah.net

*Contributed equally

Key words: spinal cord injury, autologous bone marrow-derived mesenchymal stem cells, human bone marrow-derived mesenchymal stem cells, clinical trial intractable pain. Despite current treatment strategies, including surgical decompression and fixation, the injection of neurotropic factors, anti-inflammatory medications and physical rehabilitation, satisfactory therapeutic effects remain elusive. Although a steadily accruing body of evidence points to the central nervous system possessing a means for self-repair, this capacity appears quite limited as a sole clinical approach. The complex pathology of SCI may be divided into primary and secondary injury. The primary injury is characteristically induced by mechanical damage and resultant hemorrhage. Myriad factors contributing to secondary injury include: excitatory amino acid toxicity, oxidative damage, inflammation and autoimmune response. These combined injury mechanisms, leading to glial and neuronal cell death, demyelinization and axonal degeneration, are manifested as a severe impairment in neurological function (1).

Stem cell transplantation has emerged as a possible alternative therapy for refractory nervous system conditions, including SCI $(2,3)$. Approved by the Food and Drug Administration (FDA) of the United States of America and the State Food and Drug Administration (SFDA) in China, a limited number of clinical trials concerning stem cell transplantation have, consequently, been published (4-6). Human bone marrow-derived mesenchymal stem cells (HBMSCs), identified alongside hematopoietic stem cells and possessing tremendous capacity for self-renewal and differentiation, are a type of adult stem cell that have demonstrated positive effects in the treatment of SCI (7-9). In the current study, we report on the safety and therapeutic efficacy of autologous HBMSC transplantation in 20 SCI patients treated at Wuhan Hongqiao Brain Hospital, China.

\section{Patients and methods}

Cases. A total of 20 SCI patients were enrolled in our study at the Wuhan Hongqiao Brain Hospital from 2009 to 2010. There were 13 male patients $(65 \%)$ and 7 female patients $(35 \%)$ aged 9-72 years, with an average age of 41.1 years. The maximum duration since the time of SCI was 10 years with a minimum 
Table I. Basic information of patients when admitted to hospital.

\begin{tabular}{|c|c|c|c|c|c|c|}
\hline Number & Gender & $\begin{array}{c}\text { Age } \\
\text { (years) }\end{array}$ & Injury site & Cause of injury & Time of injury & ASIA grade \\
\hline 1 & Male & 42 & T11-12 & $\begin{array}{l}\text { Acute hemorrhage } \\
\text { in the thoracic vertebrae tract }\end{array}$ & $>1$ year & A \\
\hline 2 & Male & 31 & $\mathrm{C} 5-7$ & Car accident & $>1$ year & A \\
\hline 3 & Male & 33 & L3-4 & Gunshot wound & $>1$ year & $\mathrm{C}$ \\
\hline 4 & Male & 44 & $\mathrm{~T} 8$ & Car accident & 6 months & $\mathrm{B}$ \\
\hline 5 & Female & 43 & C5-7 & Heavy blow & $>1$ year & $\mathrm{C}$ \\
\hline 6 & Male & 36 & T12-L4 & Spinal cord schistosomiasis & 6 months & A \\
\hline 7 & Female & 39 & $\mathrm{~T} 4,5$ & Hematoma outside the spinal cord & $>1$ year & $\mathrm{B}$ \\
\hline 8 & Male & 38 & $\mathrm{C} 2-6$ & Trauma & $>1$ year & $\mathrm{C}$ \\
\hline 9 & Male & 72 & L3-4 & Nerve sheath tumor with hemorrhage & 7 months & $\mathrm{C}$ \\
\hline 10 & Male & 68 & Cervical cord & Fall injury & $>1$ year & $\mathrm{C}$ \\
\hline 11 & Female & 44 & L4-5 & Trauma & 8 months & A \\
\hline 12 & Male & 56 & $\mathrm{~T} 8$ & Spinal cord atrophy & $>1$ year & $\mathrm{C}$ \\
\hline 13 & Female & 32 & $\mathrm{~L} 2-3$ & Trauma & $>1$ year & $\mathrm{B}$ \\
\hline 14 & Female & 64 & T10-12 & Car accident & $>1$ year & A \\
\hline 15 & Male & 34 & T5, T9, T10 & Car accident & $>1$ year & A \\
\hline 16 & Male & 30 & $\mathrm{~T} 12$ & Heavy blow & 3 months & A \\
\hline 17 & Female & 9 & $\mathrm{~T} 5-7$ & Myelitis & >1 year & B \\
\hline 18 & Male & 38 & $\mathrm{~T} 2-5$ & Fall injury & $>1$ year & A \\
\hline 19 & Male & 17 & S3 & Car accident & $>1$ year & $\mathrm{C}$ \\
\hline 20 & Female & 52 & $\mathrm{~T} 2$ & Trauma & $>1$ year & $\mathrm{C}$ \\
\hline
\end{tabular}

ASIA, American Spinal Injury Association.

time of 3 months (Table I). According to the American Spinal Injury Association's classification of SCI (ASIA impairment scale) there were 8 cases ( 6 males and 2 females) of grade A, 4 cases ( 1 male and 3 females) of grade B and 8 cases ( 6 males and 2 females) of grade C. Among all SCI patients, there were 4 cases involving the cervical region, 11 cases involving the thoracic region and 5 cases involving the lumbosacral region. When divided into the etiology of SCI, there were 5 cases caused by car accidents, 7 cases caused by violence, 2 cases caused by a fall and 6 cases not caused by trauma. This study was conducted in accordance with the Declaration of Helsinki and with approval from the Ethics Committee of Wuhan Hongqiao Brain Hospital. Written informed consent was obtained from all participants.

\section{Transplantation methods}

Preparation of HBMSCs. Under aseptic conditions, a puncture surgery was conducted, isolating $10 \mathrm{ml}$ bone marrow from the upper edge of the patient's iliac bone. The collected bone marrow was immediately anticoagulated by heparin and sent to the laboratory. HBMSCs were isolated and cultured in a laboratory following good manufacturing practice (GMP). Cultured cells were passaged weekly and, after 3 weeks of passage, when $1 \times 10^{8}$ cells were produced, the cells were subsequently stored in liquid nitrogen until required.

Cell transplantation. One hour prior to transplantation, the cells were resuspended in $5 \mathrm{ml}$ saline. The patients received transplanted stem cells via lumbar puncture or by computed tomography (CT)-guided injection that directly targeted the lesion sites. A total of $5 \mathrm{ml}\left(1 \times 10^{8}\right)$ BMSCs were injected into each SCI patient.

Neurological grading. Neurological grading was performed using the ASIA impairment scale as follows: Grade A, complete: no motor or sensory function is preserved in the sacral segments S4-S5; grade B, incomplete: sensory but not motor function is preserved below the neurological level and includes the sacral segments S4-S5; grade C, incomplete: motor function is preserved below the neurological level and more than half of key muscles below the neurological level have a muscle grade $<3$; grade $\mathrm{D}$, incomplete: motor function is preserved below the neurological level and at least half of the key muscles below the neurological level have a muscle grade of $\geq 3$; and grade $\mathrm{E}$, normal: motor and sensory function is normal.

All the patients were assessed for ASIA rating on the day prior to transplantation, as well as at regular intervals following treatment.

Muscle tone, abnormal motion and paresthesia. The Ashworth scale of muscle spasticity is considered a valid measure of increasing muscle tone but not of reduced muscle tone. SCI, however, may lead to either an increase or reduction in muscle tone. Consequently, in the present clinical trial, the physical characteristics of muscle changes were recorded instead of a score according to the Ashworth scale. Patients' description of 
Table II. ASIA rating before and after cell transplantation.

ASIA rating improvement 30 days after transplantation

\begin{tabular}{lccccc}
$\begin{array}{l}\text { ASIA rating before } \\
\text { transplantation }\end{array}$ & $\begin{array}{c}\text { Initial cases } \\
(\mathrm{n})\end{array}$ & 0 & 1 & 2 & $\begin{array}{c}\text { No. of cases presenting } \\
\text { improvement }\end{array}$ \\
\cline { 3 - 5 } & 8 & 4 & 3 & 1 & $4(50 \%)$ \\
A & 4 & 1 & 3 & 0 & $3(75 \%)$ \\
B & 8 & 0 & 8 & 0 & $8(100 \%)$ \\
C & 20 & $5(5 \%)$ & $14(70 \%)$ & $1(5 \%)$ & $15(75 \%)$ \\
Total & &
\end{tabular}

ASIA, American Spinal Injury Association.

abnormal motion and sensation, including zonesthesia, numbness and hyperesthesia were also reported.

Urinary and bowel function. Incontinence is a common issue for SCI patients. Assessments of bowel and bladder function are integral components of the Barthel activities of daily living (ADL) index. In order to better qualify the Barthel ADL index measures, however, these measures were subdivided. Bladder dysfunction was divided into no automatic micturition, incontinence, difficulty in urination, poor urine control and dribbling urine. Bowel dysfunction was divided into constipation, fecal incontinence and dry stools.

Pain. Pain, a common and typically severe sequelae, has tremendous impact on the daily life of SCI patients. Although the pathophysiology of pain has yet to be fully defined in SCI patients, its measure, considered integral, is classified as a separate therapeutic index. A subjective description of daily emotional state was also recorded and used for assessment post-transplantation.

Erectile dysfunction (ED). An erection may be induced physically, by reflex or psychologically (mental erection). Previous studies have shown that $60-70 \%$ of SCI patients desired sexual relations (10); however, in China, patients remained reluctant to discuss their sexual health issues due to cultural traditions. As a consequence of this cultural taboo, a full assessment of erectile issues was not completed; however, the information from the limited number of individual SCI patients willing to discuss this subject was assessed.

\section{Results}

Neurological grading by ASIA impairment scale. Our analysis of the $20 \mathrm{SCI}$ study patients receiving autologous HBMSC transplantation was notable for all patients exhibiting improvement in sensory and motor function. Of the 20 SCI study patients, 15 individuals (75\%) improved by one or two grades as measured by the ASIA impairment scale, with $14(93.3 \%)$ of those 15 patients improving by one grade and $1(6.7 \%)$ of the 15 patients improving by two grades (Table II).

As described in Table II, a lower ASIA rating prior to transplantation resulted in a slower recovery. Our data suggested that autologous HBMSC transplantation had a greater therapeutic effect in moderately injured patients than in severely injured patients. There were $14(93.3 \%)$ patients whose condition increased by one grade and 1 patient $(6.7 \%)$ whose condition increased by two grades; however, no patient's condition increased by three grades.

The prognosis of SCI patients appears to be dependent on the stage of SCI, the cause(s) of SCI and patient age. Considering the multiple disparate characteristics of our study patients, we compared the therapeutic effects of autologous HBMSC transplantation on SCI patients according to the variables in Table III.

Our clinical data, although lacking a randomized control group, was based on the statistical analysis of our SCI cohort. The data was analyzed by $\chi^{2}$ test, with $\alpha$ set at 0.05 . Limitations in data set size, however, precluded statistical significance being reached for time of injury, cause of injury, age and gender, as they relate to the efficacy of stem cell transplantation for SCI.

Assessment of urinary and bowel function. Of the 10 individuals ( $50 \%$ of the total SCI study subjects) suffering from urinary dysfunction, pre-stem cell treatment classification included: 1 patient with no automatic micturition, 4 patients with incontinence, 3 patients with difficulty in urination, 1 patient with poor urine control and 1 patient with dribbling urine. Patient recovery information is shown in Table IV. No automatic micturition is defined as urinary retention requiring catheterization. Difficulty in urination refers to laborious, non-smooth urine production. Of the ten SCI individuals suffering from urinary dysfunction, $80 \%$ (8/10) experienced post-stem cell transplantation improvement in urinary function to varying degrees.

Of the 12 individuals ( $60 \%$ of the total SCI study subjects) suffering from bowel dysfunction, pre-stem cell treatment classification included: 5 patients with constipation, 5 patients with fecal incontinence and 2 patients with dry stools. Patient recovery information is listed in Table V. Of the 12 individuals suffering from bowel dysfunction, 9 cases $(60 \%)$ experienced post-stem cell transplantation improvement in bowel function to varying degrees.

Changes of muscle tone, abnormal motion and sensation. Prior to stem cell transplantation, abnormal muscle tone was measured in 13 SCI patients, with 6 patients experiencing 
Table III. Different variables impacting the efficacy of stem cell transplantation.

\begin{tabular}{|c|c|c|c|c|}
\hline Factors & $\begin{array}{l}\text { Initial cases } \\
\text { (n) }\end{array}$ & $\begin{array}{l}\text { Cases improved } 30 \text { days after } \\
\text { cell transplantation }(n)\end{array}$ & $\begin{array}{l}\text { Improvement } \\
\text { rate }(\%)\end{array}$ & P-value \\
\hline \multicolumn{5}{|l|}{ Time since injury } \\
\hline$<1$ year & 15 & 12 & 80.0 & NS (0.56) \\
\hline$>1$ year & 5 & 3 & 60.0 & \\
\hline \multicolumn{5}{|l|}{ Cause of injury } \\
\hline Trauma & 14 & 9 & 64.3 & NS $(0.26)$ \\
\hline Non-trauma & 6 & 6 & 100 & \\
\hline \multicolumn{5}{|l|}{ Site of injury } \\
\hline Cervical vertebrae & 4 & 3 & 75.0 & NS (0.646) \\
\hline Thoracic vertebrae & 11 & 9 & 81.8 & \\
\hline Lumbosacral & 5 & 3 & 60.0 & \\
\hline \multicolumn{5}{|l|}{ Age (years) } \\
\hline$<18$ & 2 & 2 & 100 & NS (0.67) \\
\hline $18-60$ & 15 & 11 & 73.3 & \\
\hline$>60$ & 3 & 2 & 66.7 & \\
\hline \multicolumn{5}{|l|}{ Gender } \\
\hline Male & 13 & 11 & 84.6 & NS (0.29) \\
\hline Female & 7 & 4 & 57.1 & \\
\hline
\end{tabular}

NS, not significant.

Table IV. Recovery of urinary function in spinal cord injury (SCI) patients.

\begin{tabular}{lcc}
\hline Types & $\begin{array}{c}\text { Initial cases } \\
(\mathrm{n})\end{array}$ & $\begin{array}{c}\text { Cases improved } \\
\text { 30 days after cell } \\
\text { transplantation (n) }\end{array}$ \\
\hline No automatic micturition & 1 & 1 \\
Incontinence & 4 & 3 \\
Difficulty in urination & 3 & 2 \\
Poor urine control & 1 & 1 \\
Dribbling urine & 1 & 1 \\
\hline
\end{tabular}

reduced muscle tone and 7 patients experiencing increased muscle tone. Patient recovery information is listed in Table VI.

As normal muscle tone is the basis for coordinated human motor function, perturbations in natural muscle tone manifest as problems in daily functioning. As previously noted, the Ashworth scale of muscle spasticity is a common standard used for the assessment of increasing muscle tone. Table VI shows that HBMSC transplantation had a greater effect on qualitatively improving increased rather than reduced muscle tone issues in SCI patients.

Abnormal, non-autonomic movement occurred in the two lower extremities in 1 of the 20 patients following SCI. Six patients complained of abnormal sensations, including zonesthesia, electric shock-like sensation, numbness and hyperesthesia (Table VII).
Table V. Recovery of bowel function in spinal cord injury (SCI) patients.

\begin{tabular}{lcc}
\hline Types & $\begin{array}{c}\text { Initial cases } \\
\text { (n) }\end{array}$ & $\begin{array}{c}\text { Cases improved } \\
\text { 30 days after cell } \\
\text { transplantation (n) }\end{array}$ \\
\hline Constipation & 5 & 4 \\
Fecal incontinence & 5 & 3 \\
Dry stools & 2 & 2 \\
\hline
\end{tabular}

Although improvement in abnormal motion was not noted, all the patients with zonesthesia improved following cell transplantation, with certain patients experiencing complete resolution of this abnormal sensation.

Assessment of pain and ED. Neuralgia, particularly in the extremities, is a well-documented phenomenon in SCI patients (11). Four of the patients in the current clinical trial reported neuralgia with three patients characterizing extremity pain and one patient with headache; this neuralgia, as noted, was greatly improved following HBMSC transplantation (Table VIII).

A previous study reported that $\sim 25 \%$ of SCI patients experience ED (12). As is customary for traditional cultures, discussion of sexuality is considered a taboo. For this reason only two of our male SCI patients were willing to discuss erectile issues; of the two male SCI patients, one experienced improvement in penile sensitivity and tumescence following HBMSC transplantation. 
Table VI. Recovery of abnormal muscle tone in spinal cord injury (SCI) patients.

\begin{tabular}{lccc}
\hline $\begin{array}{l}\text { Muscle } \\
\text { tone }\end{array}$ & $\begin{array}{c}\text { Initial cases } \\
(\mathrm{n})\end{array}$ & $\begin{array}{c}\text { Cases improved 30 days after } \\
\text { cell transplantation }(\mathrm{n})\end{array}$ & $\begin{array}{c}\text { Improvement } \\
\text { rate }(\%)\end{array}$ \\
\hline Increase & 7 & 7 & 100 \\
Decrease & 6 & 2 & 33.3 \\
\hline
\end{tabular}

Table VII. Abnormal motion and sensation.

\begin{tabular}{lcc}
\hline & $\begin{array}{c}\text { Initial cases } \\
\text { Types }\end{array}$ & $\begin{array}{c}\text { Cases improved } \\
30 \text { days after cell } \\
\text { transplantation (n) }\end{array}$ \\
\hline $\begin{array}{l}\text { Abnormal motion } \\
\text { Frequent non-autonomic }\end{array}$ & 1 & 0 \\
Abnormal sensation & & \\
Zonesthesia & 3 & 3 \\
Numbness & 1 & 0 \\
Hyperesthesia & 1 & 0 \\
Electric shock-like & 1 & 0 \\
\hline
\end{tabular}

\section{Discussion}

A series of animal experiments (13-17) and clinical trials (18-23) have previously demonstrated that stem cells have beneficial effects for SCI. In the current clinical trial we report on the use of autologous HBMSC transplantation in patients with SCI. Our study demonstrates that multiple sequelae associated with SCI, including sensory and motor dysfunction, abnormal muscle tone, urinary and bowel functional disorders, insufferable pain and ED, may improve significantly following stem cell transplantation.

A study by Yoon et al using HBMSC transplantation for the treatment of SCI (18) demonstrated that $29.5 \%$ of patients in the acute stage ( $<2$ weeks) experienced an improvement in ASIA impairment rating from grade $\mathrm{A}$ to either B or C. Additionally, $33.3 \%$ of patients in the subacute stage (2-8 weeks) experienced an improvement in ASIA impairment rating from grade A to either B or $\mathrm{C}$; while no improvement in ASIA impairment scale occurred in the chronic ( $>8$ weeks) group. However, in the current study, $75 \%$ of SCI patients experienced an improvement in ASIA rating, with the majority of these patients receiving cell transplantation half a year after SCI, with the exception of one patient who was in the subacute (3 month) SCI stage. Cell therapy appears to have been more beneficial for the patients in the current study than for those in the study by Yoon et al, as the patients in our group were of three different ASIA grades prior to cell transplantation whereas all patients in the other study were of grade A. In theory, the higher the grade, the greater the improvement; however, our data suggests an inconsistency with this theory. In addition, while our study followed-up patients for one month, Yoon et al continued for 10.4 months. A question, hence, arises as to whether the therapeutic effects of stem cell therapy decline over time and, if verified, what the origin of this phenomenon may be. Once
Table VIII. Recovery of pain and erectile dysfunction (ED) in spinal cord injury (SCI) patients.

\begin{tabular}{lcc}
\hline & $\begin{array}{c}\text { Initial cases } \\
\text { (n) }\end{array}$ & $\begin{array}{c}\text { Cases improved } \\
\text { 30 days after cell } \\
\text { transplantation (n) }\end{array}$ \\
\hline Pain & & \\
Extremities pain & 3 & 3 \\
Headache & 1 & 1 \\
ED & 2 & 1 \\
\hline
\end{tabular}

these questions are answered, the benefits of stem cell therapy and its applications may be appreciated.

Abnormal muscle tone in SCI patients includes increases and reductions in muscle tone. For SCI patients, lesion location plays an integral role in the subsequent quality of muscle tone. A lesion in the anterior root or posterior funiculus of the spinal cord leads to reduced muscle tone, while a lesion in the pyramidal tracts causes an increase in muscle tone. In our study all SCI patients with increased muscle tone experienced improvement, while only one-third of SCI patients with reduced muscle tone experienced an improvement. Although statistical significance was achieved with regard to this measure $(\mathrm{P}=0.021)$, limitations in sample size make it unclear whether stem cell therapy impacts either increased or reduced muscle tone more substantially.

Normal urinary and bowel function make a significant contribution to quality of life. Of the 10 SCI patients $(50 \%$ of total) reporting urinary dysfunction, $80 \%$ experienced improved micturition following stem cell transplantation. In opposition to the study by Kishk et al (23), where no patients experienced complete recovery in urinary function, two patients in our study experienced a return to normal function; one with urinary incontinence and the other with dribbling urine prior to stem cell therapy. Of the 12 patients $(60 \%$ of total) with bowel dysfunction, $75 \%$ experienced an improvement in function following stem cell therapy; this result is commensurate with the results from the study by Kishk et al. In addition to aiding the functional integrity of autonomic nerves for normal urinary and bowel function, the return of intestinal secretions following stem cell transplantation may have ameliorated a significant factor in the bowel dysfunction in certain patients.

Pain is a common and severe condition associated with SCI. In the survey by Wrigley et al (11), two-thirds of SCI patients suffered from pain that was classified based on the level of injury; while one third of patients suffered from pain, 
which occurred below the level of injury and was more severe in nature and considered more difficult to treat. Methods used to combat the pain included psychotherapy and the use of anxiolytic and antidepressant medications. However, while two patients from the study demonstrated improvement, further research of stem cell therapy is required to determine the impact of this therapeutic modality on pain.

There were few reports of abnormal sensation brought on by SCI in patients. Of the 6 patients endorsing either zonesthesia, electric shock-like sensations, numbness or hyperesthesia in our study, only the symptom of zonesthesia improved with stem cell therapy; the pathophysiology behind this symptom remains unclear.

Due to the limited number of patients and lack of a control group, we were not able to relate the efficiency of stem cell transplantation to age or gender, nor to time or cause of injury. To obtain statistical significance regarding these variables, a larger number of patients and a control group are warranted.

The most common adverse events in the present study were fever and headache, which disappeared after 1-2 days without any treatment. No patients developed severe adverse effects, further demonstrating the safety of autologous HBMSC transplantation. However, due to the lack of a long-term follow-up, the formation of tumors should not be excluded. To completely assess the possible tumorigenic risk, a long-term follow-up study would be prudent.

Although limitations exist, HBMSC transplantation has demonstrated its effectiveness for the treatment of SCI. The majority of our patients clearly benefited from transplantation with notable improvements in sensory, motor and autonomic function. The mechanisms by which stem cells benefit SCI patients, however, are not fully clear. Currently, the mechanisms by which stem cells are believed to repair damaged tissue include the secretion of neurotrophic factors, the ability to re-wrap injured nerve fibers suffering demyelization and the formation of neural circuitry by transplanted cells that are able to differentiate into neurons (24-30). However, despite steadily accruing evidence in support of the therapeutic benefits of stem cell transplantation, universal consensus regarding the mechanisms of action does not yet exist. Additional studies of autologous HBMSC transplantation for the treatment of SCI remain a critical pursuit.

\section{Acknowledgements}

The authors are grateful to all the individuals participating in this study. The study would not have been possible without the cooperation of the patients and their families, the donors and the assistance of the doctors, nurses and physical therapists at Wuhan Hongqiao Brain Hospital.

\section{References}

1. Barnabé-Heider F and Frisén J: Stem cells for spinal cord repair. Cell Stem Cell 3: 16-24, 2008.

2. Lim PA and Tow AM: Recovery and regeneration after spinal cord injury: a review and summary of recent literature. Ann Acad Med Singapore 36: 49-57, 2007.

3. Goldman S: Stem and progenitor cell-based therapy of the human central nervous system. Nat Biotechnol 23: 862-871, 2005.

4. Harting MT, Baumgartner JE, Worth LL, et al: Cell therapies for traumatic brain injury. Neurosurg Focus 24: E18, 2008.
5. Park HC, Shim YS, Ha Y, et al: Treatment of complete spinal cord injury patients by autologous bone marrow cell transplantation and administration of granulocyte-macrophage colony stimulating factor. Tissue Eng 11: 913-922, 2005.

6. Zhao CH (ed): Theory, Technology and Clinical of Stem Cells. Beijing: Chemical Industry Press, Beijing, 2006.

7. Satake K, Lou J and Lenke LG: Migration of mesenchymal stem cells through cerebrospinal fluid into injured spinal cord tissue. Spine (Phila Pa 1976) 29: 1971-1979, 2004.

8. Koda M, Nishio Y, Kamada T, et al: Granulocyte colony stimulating (G-CSF) mobilizes bone marrow-derived cells into injured spinal cord and promotes functional recovery after compression-induced spinal cord injury in mice. Brain Res 1149: 223-231, 2007.

9. Parr AM, Kulbatski I, Zahir T, et al: Transplanted spinal cord-derived neural stem/progenitor cells promote early functional recovery after rat spinal cord injury. Neuroscience 155: 760-770, 2008 .

10. Julia PE and Othman AS: Barriers to sexual activity: counselling spinal cord injured women in Malaysia. Spinal Cord 49: 791-794, 2011.

11. Wrigley PJ, Press SR, Gustin SM, et al: Neuropathic pain and primary somatosensory cortex reorganization following spinal cord injury. Pain 141: 52-59, 2009.

12. Kim HS, Kim NH, Lee HM, Park HW, Ha JW and Park JO: Sexual dysfunction in men with paraparesis in lumbar burst fractures. Spine (Phila Pa 1976) 25: 2187-2190, 2000.

13. Park WB, Kim SY, Lee SH, Kim HW, Park JS and Hyun JK: The effect of mesenchymal stem cell transplantation on the recovery of bladder and hindlimb function after spinal cord contusion in rats. BMC Neurosci 11: 119, 2010.

14. Novikova LN, Brohlin M, Kingham PJ, Novikov LN and Wiberg M: Neuroprotective and growth-promoting effects of bone marrow stromal cells after cervical spinal cord injury in adult rats. Cytotherapy 13: 873-887, 2011.

15. Mothe AJ, Bozkurt G, Catapano J, et al: Intrathecal transplantation of stem cells by lumbar puncture for thoracic spinal cord injury in the rat. Spinal Cord 49: 967-973, 2011.

16. Nishida H, Nakayama M, Tanaka H, et al: Evaluation of transplantation of autologous bone marrow stromal cells into the cerebrospinal fluid for treatment of chronic spinal cord injury in dogs. Am J Vet Res 72: 1118-1123, 2011.

17. Deng YB, Yuan QT, Liu XG, et al: Functional recovery after rhesus monkey spinal cord injury by transplantation of bone marrow mesenchymal-stem cell-derived neurons. Chin Med J (Engl) 118: 1533-1541, 2005.

18. Yoon SH, Shim YS, Park YH, et al: Complete spinal cord injury treatment using autologous bone marrow cell transplantation and bone marrow stimulation with granulocyte macrophage-colony stimulating factor: Phase I/II clinical trial. Stem Cells 25: 2066-2073, 2007.

19. Geffner LF, Santacruz P, Izurieta M, et al: Administration of autologous bone marrow stem cells into spinal cord injury patients via multiple routes is safe and improves their quality of life: comprehensive case studies. Cell Transplant 17: 1277-1293, 2008.

20. Kumar AA, Kumar SR, Narayanan R, et al: Autologous bone marrow derived mononuclear cell therapy for spinal cord injury: A phase I/II clinical safety and primary efficacy data. Exp Clin Transplant 7: 241-248, 2009.

21. Chernykh ER, Stupak VV, Muradov GM, et al: Application of autologous bone marrow stem cells in the therapy of spinal cord injury patients. Bull Exp Biol Med 143: 543-547, 2007.

22. Saito F, Nakatani T, Iwase M, et al: Spinal cord injury treatment with intrathecal autologous bone marrow stromal cell transplantation: the first clinical trial case report. J Trauma 64: 53-59, 2008.

23. Kishk NA, Gabr H, Hamdy S, et al: Case control series of intrathecal autologous bone marrow mesenchymal stem cell therapy for chronic spinal cord injury. Neurorehabil Neural Repair 24: 702-708, 2010.

24. Sharp J, Frame J, Siegenthaler M, et al: Human embryonic stem cell-derived oligodendrocyte progenitor cell transplants improve recovery after cervical spinal cord injury. Stem Cells 28: 152-163, 2010.

25. Hwang DH, Kim BG, Kim EJ, et al: Transplantation of human neural stem cells transduced with Olig2 transcription factor improves locomotor recovery and enhances myelination in the white matter of rat spinal cord following contusive injury. BMC Neurosci 10: 117, 2009. 
26. Keirstead HS, Nistor G, Bernal G, et al: Human embryonic stem cell-derived oligodendrocyte progenitor cell transplants remyelinate and restore locomotion after spinal cord injury. J Neurosci 25: 4694-4705, 2005.

27. Vaquero J, Zurita M, Oya S and Santos M: Cell therapy using bone marrow stromal cells in chronic paraplegic rats: systemic or local administration? Neurosci Lett 398: 129-134, 2006.

28. Akiyama Y, Radtke C and Kocsis JD: Remyelination of the rat spinal cord by transplantation of identified bone marrow stromal cells. J Neurosci 22: 6623-6630, 2002.
29. Park HW, Lim MJ, Jung H, et al: Human mesenchymal stem cell-derived Schwann cell-like cells exhibit neurotrophic effects, via distinct growth factor production, in a model of spinal cord injury. Glia 58: 1118-1132, 2010.

30. Weimann JM, Johansson CB, Trejo A and Blau HM: Stable reprogrammed heterokaryons form spontaneously in Purkinje neurons after bone marrow transplant. Nat Cell Biol 5: 959-966, 2003. 